\title{
Chronobiology and Metabolism: Is Ketogenic Diet Able to Influence Circadian Rhythm?
}

\author{
Elena Gangitano ${ }^{1,2 *}$, Lucio Gnessi ${ }^{1}$, Andrea Lenzi' and David Ray ${ }^{2,3}$ \\ ${ }^{1}$ Department of Experimental Medicine, Sapienza University of Rome, Rome, Italy, ${ }^{2}$ Oxford Centre for Diabetes, \\ Endocrinology and Metabolism, University of Oxford, Oxford, United Kingdom, ${ }^{3}$ NIHR Oxford Biomedical Research Centre, \\ John Radcliffe Hospital, Oxford, United Kingdom
}

\section{OPEN ACCESS}

Edited by: Zoltan Sarnyai, James Cook University, Australia

Reviewed by: David Whitmore, James Cook University, Australia Paul Guest, University of Cambridge, United Kingdom

*Correspondence: Elena Gangitano elena.gangitano@uniroma1.it

Specialty section:

This article was submitted to Neuroenergetics, Nutrition and Brain Health,

a section of the journal Frontiers in Neuroscience

Received: 12 August 2021 Accepted: 19 October 2021 Published: 08 November 2021

Citation:

Gangitano E, Gnessi L, Lenzi A and Ray D (2021) Chronobiology and Metabolism: Is Ketogenic Diet Able to Influence Circadian Rhythm?

Front. Neurosci. 15:756970. doi: 10.3389/fnins.2021.756970
Circadian rhythms underpin most physiological processes, including energy metabolism. The core circadian clock consists of a transcription-translation negative feedback loop, and is synchronized to light-dark cycles by virtue of light input from the retina, to the central clock in the suprachiasmatic nucleus in the hypothalamus. All cells in the body have circadian oscillators which are entrained to the central clock by neural and humoral signals. In addition to light entrainment of the central clock in the brain, it now emerges that other stimuli can drive circadian clock function in peripheral tissues, the major one being food. This can then drive the liver clock to be misaligned with the central brain clock, a situation of internal misalignment with metabolic disease consequences. Such misalignment is prevalent, with shift workers making up $20 \%$ of the working population. The effects of diet composition on the clock are not completely clarified yet. High-fat diet and fasting influence circadian expression of clock genes, inducing phase-advance and phase-delay in animal models. Ketogenic diet (KD) is able to induce a metabolic switch from carbohydrate to fatty acid oxidation, miming a fasting state. In recent years, some animal studies have been conducted to investigate the ability of the KD to modify circadian gene expression, and demonstrated that the KD alters circadian rhythm and induces a rearrangement of metabolic gene expression. These findings may lead to new approaches to obesity and metabolic pathologies treatment.

Keywords: chronobiology, circadian rhythm, circadian misalignment, metabolism, ketogenic diet (KD)

\section{INTRODUCTION}

\section{The Clock Machinery and the Circadian Rhythm}

In mammalians many genes exhibit daily fluctuations in their expression levels, configuring a circadian rhythm of approximately $24 \mathrm{~h}$. This circadian oscillation is internally generated (Ko and Takahashi, 2006; Dibner et al., 2010) and driven by clock machinery.

At a cellular level the circadian clock is constituted by core clock genes, including CLOCK, $B M A L 1, P E R$, and $C R Y$, that are connected by transcriptional-translational feedback loops (Albrecht, 2012). The feedback loop produces oscillations in gene expression, associated with circadian changes in chromatin architecture, mRNA processing, and protein activity and turnover (Eckel-Mahan et al., 2013). CLOCK and BMAL1 are the core heterodimeric transcription factor 
which drives expression of $P E R$, and $C R Y$ genes, and the REVERB genes, by binding to conserved DNA sequences termed $\mathrm{E}$ boxes. BMAL1/CLOCK transactivation of target genes such as PER and $C R Y$, which are part of the negative feedback loop, results in the accumulation of their protein products PER and CRY proteins. PER and CRY act together to repress the activity of the BMAL1/CLOCK heterodimer, thus forming one negative feedback loop. CLOCK:BMAL1 also activate the transcription of the nuclear receptors REV-ERB and RORA, whose proteins compete to bind the BMAL1 promoter at a ROR binding site. ROR activates $B M A L 1$ transcription, while REV-ERBs represses it (Ko and Takahashi, 2006). Thus there are two negative feedback loops that together confer a robust $24 \mathrm{~h}$-period oscillator.

The circadian clock synchronizes internal metabolism with the environment, interacting with light-dark and feedingfasting state (Panda, 2016). The central clock is set in the suprachiasmatic nucleus $(\mathrm{SCN})$ of the anterior hypothalamus, and it is synchronized to the external light-dark cycle by direct neural input from the retina. The central clock is set at the top of the hierarchy of the circadian oscillation and drives the peripheral clocks oscillation in a synchronized way. In peripheral tissues all cells also have a circadian oscillator. However, in the absence of light-sensing these clocks are kept synchronized by neural and humoral outputs from the SCN. In addition, peripheral clocks will entrain to feeding cycles, a phenomenon termed food entrainment (see Figure 1). It is possible to drive the circadian phase of the liver to be in anti-phase to that of the SCN by restricting feeding to the conventional rest period (Damiola et al., 2000); night in humans. Other metabolic tissues, as adipose tissue, have their own clocks, which are normally kept synchronized with the liver clock and the other peripheral clocks.

The clock machinery controls physiology processes by regulating the expression of hundreds of metabolic genes, involved in the rate-limiting steps of fundamental metabolic pathways. Therefore it orchestrates metabolism in cycles of $24 \mathrm{~h}$, and increases metabolic efficiency thanks to anticipatory responses to the feeding-fasting cycles and temporal separation of opposite metabolic processes (Panda, 2016; Poggiogalle et al., 2018; Reinke and Asher, 2019).

Peripheral tissues are implicated in the metabolic balance of the individual. Among them, the liver plays a key role in major enzymatic processes for glucose and fat metabolism. Therefore, the SCN-liver axis for circadian rhythm is probably the most important connection between the central clock and the peripheral clocks.

\section{The Ketogenic Diet}

Ketogenic Diet (KD) is a low-carbohydrate, normo-protein diet, characterized by an overproduction of the ketone bodies acetone, acetoacetate and $\beta$-hydroxyl-butyrate $(\beta \mathrm{OHB})$. KD induces a metabolic switch from carbohydrate to fatty acid oxidation, so that fat is the principal energy source. During a KD, fatty acid oxidation, ketogenesis and gluconeogenesis are upregulated and glycolysis and de novo lipogenesis are markedly reduced (Tognini et al., 2017), and these metabolic modifications are similar to those physiologically seen during fasting or significative caloric restriction (Tognini et al., 2017). Ketone bodies are not only an energy source, directed from the liver to the periphery during fasting conditions and exercise, but also important signaling molecules (Newman and Verdin, 2014). $\beta$ OHB can bind to G-protein-coupled receptors for short-chain fatty acids on the cell surface, reducing lipolysis (Taggart et al., 2005) and sympathetic activity (Kimura et al., 2011). On the other hand, short-chain fatty acids promote sympathetic nervous system activation (Kimura et al., 2011). Ketone bodies are also able to inhibit histone deacetylase and induce hyperacetylation, similarly to what happens in fasting conditions, and determine changes in gene expression (Shimazu et al., 2013; Newman and Verdin, 2014). In addition, ketone bodies are also implicated in the mechanism of food anticipation (Chavan et al., 2016; Chaix and Panda, 2016).

In clinical settings, there are different kinds of $\mathrm{KD}$, which differ in the degree of calorie restriction and macronutrient composition (Kirkpatrick et al., 2019). The KD was originally proposed for the treatment of refractory epilepsy in children and over time has been proven to be particularly effective in treating morbid obesity and metabolic diseases, providing a relatively fast weight loss with concomitant preservation of muscle mass. KD characterized by a very low-calorie content (VLCKD) is actually considered for prescription in severe obesity and obesity complicated by type 2 diabetes, hypertriglyceridemia and/or hypertension (Caprio et al., 2019), that configure a picture of severely metabolically compromised patients. There are some contraindications to $\mathrm{KD}$ prescription. In particular, according to the Italian consensus on VLCKD, it is absolutely contraindicated in some patients, as patients with organ failure (respiratory failure, kidney failure and moderate-to-severe chronic kidney disease, hepatic failure), some cardiovascular diseases (heart failure, unstable angina, cardiac arrhythmias and recent stroke or myocardial infarction), severe infections, type 1 diabetes mellitus, beta-cell failure in type 2 diabetes mellitus and therapy with sodium/glucose cotransporter-2 inhibitors. Moreover, VLCKD is contraindicated in frail elderly patients, during pregnancy and breastfeeding, in the peri-operative period and in case of concomitant psychiatric conditions (severe mental illnesses, eating disorders, alcohol and substance abuse) (Caprio et al., 2019).

Ketogenic diet has been recently proposed as an adjuvant treatment for other illnesses (Paoli et al., 2013), as migraine (Di Lorenzo et al., 2019), polycystic ovary syndrome (Paoli et al., 2020), cancer (Chung and Park, 2017), neurodegenerative diseases (Wlodarek, 2019) and even COVID-19 (Gangitano et al., 2021; Sukkar et al., 2021). Question remains about the relative impact of fat content, protein content, and overall calorie content.

\section{CLOCK DESYNCHRONIZATION AND METABOLISM: THE IMPORTANCE OF THE TIMING OF FOOD INTAKE, FASTING AND SLEEP}

Internal misalignment between the phase of the circadian cycle in the central clock and that of the peripheral clocks, results 


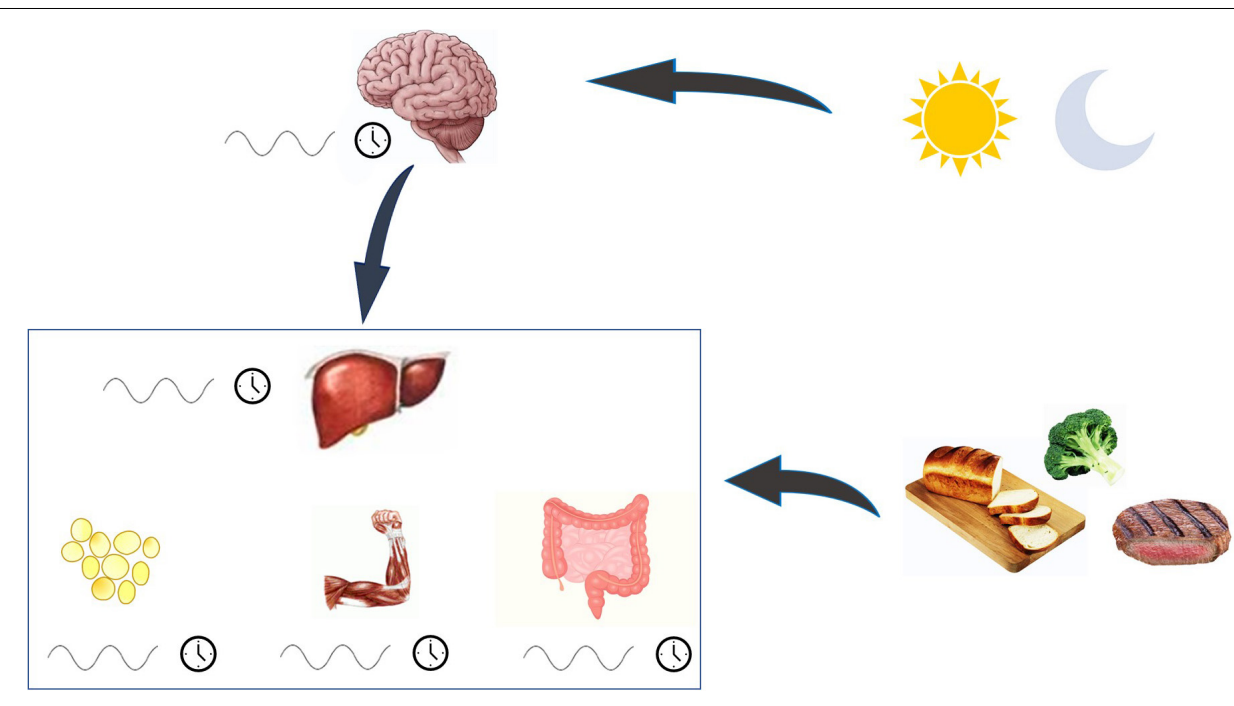

FIGURE 1 | The regulation of circadian rhythm. Biological rhythm is under the control of the central clock, set in the suprachiasmatic nucleus. This clock integrates its internal rhythm with the alternance of light and dark, and orchestrates the rhythm of peripheral clocks, which are directly entrained also by food. When all clocks are synchronized, metabolism functioning is preserved.

in disorder amongst the organs implicated in metabolism, and behavior, and may drive the onset of disease. Indeed, this prevalent phenomenon occurs in shift workers who have an increased risk of obesity and type 2 diabetes (see Figure 2).

Clock-mutant mice, with preserved rhythmicity in the suprachiasmatic nucleus and pineal gland, and arrhythmic clock genes expression in liver and skeletal muscle, are metabolically impaired, with altered glucose tolerance and insulin secretion, and altered expression of molecular determinants of metabolic homeostasis in liver and skeletal muscle, in comparison with wild-type mice (Kennaway et al., 2007).

Eating against the clock drives internal desynchronization and, among peripheral clocks, the hepatic clock is particularly sensitive to feeding time information (Damiola et al., 2000; Eckel-Mahan et al., 2013). Studies in mice reveal that animals fed during the resting phase gain weight, develop abdominal obesity, metabolic alterations (Salgado-delgado et al., 2010; Yasumoto et al., 2016), increased hepatic fat accumulation, hyperphagia and are less physically active (Yasumoto et al., 2016). These metabolically impaired animals develop alterations in the circadian expression of clock genes (Turek et al., 2005; Salgado-Delgado et al., 2013; Yasumoto et al., 2016), confirming the strict association between circadian rhythm and metabolism. Feeding during the resting phase leads to desynchronization among liver and skeletal muscle peripheral clocks (Yasumoto et al., 2016), desynchronization among liver clock genes and metabolic genes expression (Salgado-Delgado et al., 2013) and alteration of the expression of glucose and lipid metabolism-related genes (Yasumoto et al., 2016). The disruption of circadian rhythm selectively hits peripheral clocks, as the central clock is only affected by light information (Damiola et al., 2000).

By contrast, feeding mice during the active phase prevents metabolic alterations and clock genes disruption, despite the night-shift work (Salgado-delgado et al., 2010) and the diet composition (Hatori et al., 2012). Studies on mice fed HighFat (HF) diet with time-restricted access to food of $8 \mathrm{~h} /$ day, during the natural feeding time, in comparison with mice fed HF ad libitum, showed that, given the same caloric intake, weight gain and metabolic disturbances were restricted to the adlib fed animals, and that time-restricted feeding prevented the metabolic consequences of an unhealthy diet (Hatori et al., 2012). In fact, restricting food consumption to the active phase led to increased amplitude of the circadian oscillation in peripheral tissues and it was not associated to the development of obesity, hyperinsulinemia and hepatic steatosis (Hatori et al., 2012; Bae et al., 2019).

Intermittent fasting, with food given every other day, has been shown to be able to abolish the circadian expression of most clock genes, while Clock and Per2 were expressed with a decreased amplitude and a phase-advance in mouse liver (Froy et al., 2009). On the contrary, intermittent fasting was able to restore circadian rhythms and clock gene expression amplitude that was disrupted by abnormal light cycles, including cycles with a period of less than 24 h (Froy et al., 2009).

In mice without a central clock and subject to environmental disruption by being in constant darkness there is the complete disintegration of consolidated sleep-wake, and activity rhythms, with loss of feed/fasted cycles. Time-restricted food availability was sufficient to restore circadian organization to the animals' behavior, and energy metabolism with normalization of body weight and glucose metabolism (Kolbe et al., 2019). Therefore, intermittent fasting seems to be able to affect circadian rhythm differently, depending on the time of food availability (Froy et al., 2009). Furthermore, complete fasting modulates the circadian rhythm by attenuating clock gene oscillation amplitude and also induces a circadian phase advance (Barnea et al., 2009). A simultaneous shift in feeding schedule and light and dark 


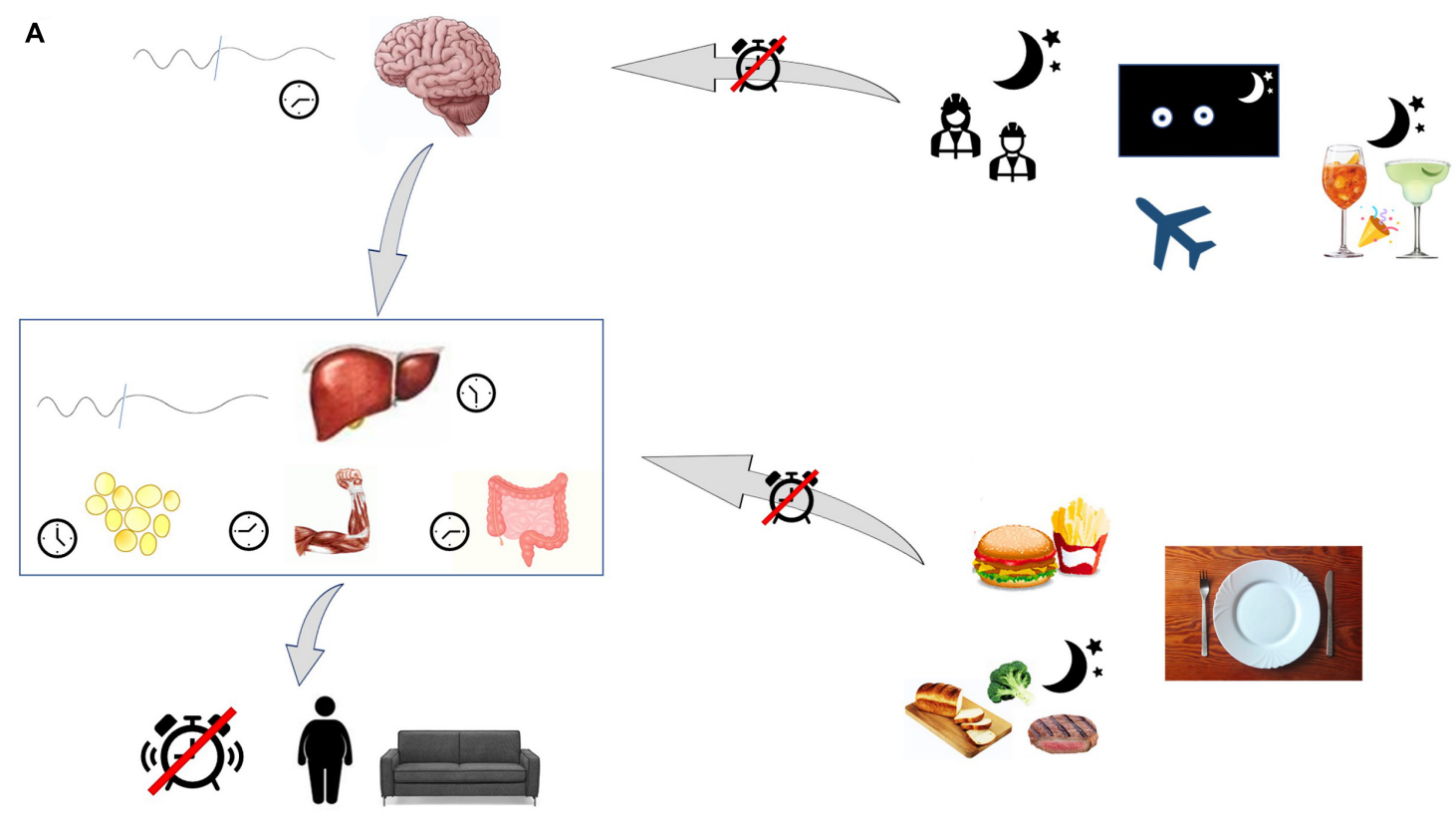

B

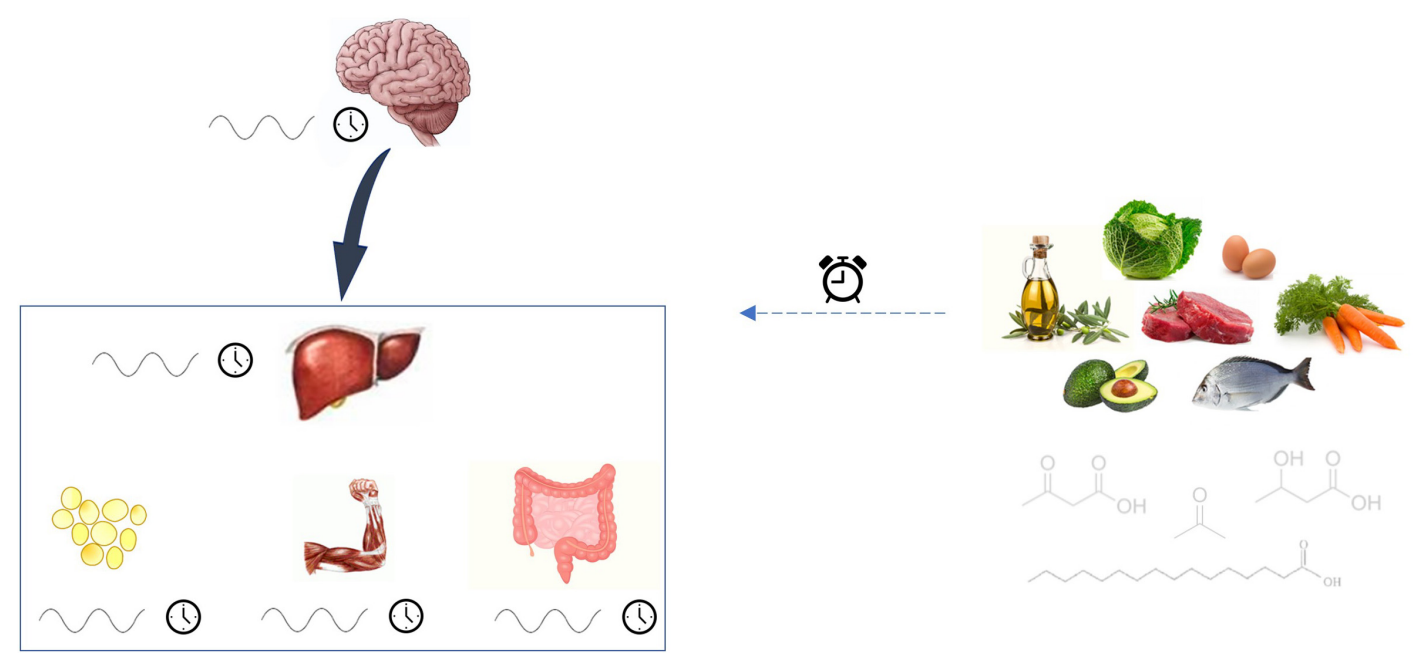

FIGURE 2 | Circadian rhythm disruption. (A) Many external factors may disturb circadian rhythmicity. Shift-work, jet-lag and social jet-lag alter the exposition to light and dark cycles. Also lack of sleep and low-quality sleep are able to disrupt central clock rhythm. Consumption of high-fat foods, as well as eating at night-time and fasting, have disruptive effects on peripheral clocks, which are led out of phase. Clocks desynchronization favors the onset of metabolic disturbances. (B) Ketogenic diet, probably through its peculiar molecules fatty acids and ketone bodies, may play a role in influencing circadian rhythm.

cycle has been proposed to facilitate circadian resetting in animal models (Wu et al., 2010).

In humans, the key role of the timing of food intake is confirmed by the fact that late eating is associated with increased BMI (Mchill et al., 2017, 2019), reduced insulin sensitivity and reduced effects of weight-loss strategies (Dashti et al., 2021). Breakfast consumption has been proven to affect clock and clock-controlled gene expression, and skipping breakfast alters core clock gene expression and also increases the postprandial glycemic response in both healthy and type 2 diabetes patients (Jakubowicz et al., 2017).

In people with sleep deprivation, typical of shift-workers, there is a preference for high-fat and sweet foods (Cain et al., 2015; Simon et al., 2015), and an association, which may be causal, with weight gain (Van Den Berg et al., 2008; Chaput et al., 2014) and development of obesity (Potter et al., 2017) and metabolic 
disturbances, as altered glucose tolerance (Grant et al., 2017; McHill and Wright, 2017). Even sleep fragmentation is associated with obesity (Van Den Berg et al., 2008; Mezick et al., 2014) and insulin resistance (McHill and Wright, 2017). A possible strategy to mitigate the risks of shiftwork is to avoid eating against the clock (Grant et al., 2017).

Social jet lag is a recently emerged phenomenon related to the shift of social life toward nocturnal hours, so that people meet each other and eventually eat and/or drink during hours normally dedicated to rest and sleep. It is characterized by a misalignment between social life and biological rhythms, configures a situation similar to the classic jet lag, and, like long flights, leads to altered circadian rhythm and metabolic consequences long-term (Almoosawi et al., 2018; Mathew et al., 2020).

\section{CLOCK DESYNCHRONIZATION AND METABOLISM: THE IMPORTANCE OF DIET COMPOSITION}

Diet composition can influence circadian clock activity, because feeding-derived metabolites play a role in regulating cellular rhythmicity (Eckel-Mahan et al., 2013), but the mechanisms through which nutrition influences the circadian metabolome and consequently circadian rhythm have not yet been elucidated.

Some animal studies show that HF diet is able to disrupt circadian rhythm (Kohsaka et al., 2007; Barnea et al., 2010), inducing a phase shift (Barnea et al., 2009, 2010; Eckel-Mahan et al., 2013) and a loss of synchronization of gene expression among liver and fat tissue (Kohsaka et al., 2007). Kohsaka et al. (2007) fed mice with a regular chow or an HF diet for 6 weeks. After 7 days, before a significant weight gain, mice fed with $\mathrm{HF}$ diet reduced their overall physical activity and increased the food intake during the resting period. Core clock gene expression was not affected in the hypothalamus, but the amplitude of Clock and Bmal1 expression was attenuated in fat and liver. Moreover, a loss of synchrony of gene expression of nuclear receptors and metabolic regulators was observed among peripheral tissues.

Barnea et al. (2009, 2010) studied mice fed an HF diet or a low-fat (LF) diet for 7 weeks, followed by a day of fasting. Clock genes oscillated in the liver, muscle and white adipose tissue (WAT), but fasting in the LF diet group led to an attenuation of clock gene amplitude. Fasting caused a circadian phase advance, but in contrast an HF diet induced a phase delay in circadian clock genes and resulting disruption of the circadian rhythmicity of the adiponectin component cascade. Diet-induced disruption in the circadian expression of adiponectin signaling components may result from metabolite regulation of peroxisome proliferator-activated receptors $\alpha$ and $\gamma(\operatorname{PPAR} \alpha$ and PPAR $\gamma)$ and MAMPK. These signaling cascades may allow feeding time to affect core circadian clock function. In turn, disruption of the clock may result in aberrant coordination of adiponectin synthesis and processing. This alteration in adiponectin signaling may be related to the development of metabolic impairment and the disruption of other clock-controlled mechanisms, as blood pressure and sleep/wake cycle, associated with metabolic syndrome.

Eckel-Mahan et al. (2013) studied mice and showed that diet composition itself is able to reprogram the clock. In particular, an HF diet profoundly reorganizes specific metabolic pathways, with a widespread remodeling of the liver clock, ablates some transcript and metabolite oscillations, generates new oscillating transcripts and, in contrast to Barnea (Barnea et al., 2009, 2010), observed that it induces a phase advance for many metabolites and oscillating transcripts. These changes are maintained on the diet and are reversible. In addition, Eckel-Mahan identified new oscillating gene transcripts in the liver that were only seen on the HF diet, while they observed that some typically oscillating genes lost a circadian signature. HF reorganizes coordinated oscillation of transcripts and metabolites through shifted CLOCK: BMAL1 chromatin recruitment and cyclic activation of surrogate pathways through the transcription factor PPAR $\gamma$. Clock and Bmal1 transcription, protein levels and their phosphorylation were unaltered in livers of HF-diet fed mice. Three days of HF diet were enough to initiate the reprogramming of the circadian clock, confirming the results of Kohsaka (Kohsaka et al., 2007) that an HF diet is able to alter circadian rhythm and behavioral activity independently from weight gain. Two weeks of normal chow were able to restore the circadian clock, proving that the transcriptional and epigenetic modifications induced by the HF diet are reversible.

\section{CIRCADIAN RHYTHM AND KETOGENIC DIET}

The effects of a ketogenic diet on clock gene expression have been recently investigated in mouse models, and these studies showed that $\mathrm{KD}$ is able to influence circadian rhythm.

Oishi et al. $(2009,2013)$ fed mice with KD or normal chow for 2 weeks, and observed that KD induced a phase-advance in peripheral clocks and behavioral activity, despite a maintained light-dark cycle and feeding ad libitum. The phase-advance effect was greater in the heart, kidney, and adipose tissue, than in the liver. Moreover, some clock genes showed a higher amplitude of their expression in the liver, while their amplitude in the heart was substantially unaffected (Oishi et al., 2013). The authors hypothesized that this robust oscillation may be related to CIRBP expression, a protein linked to hypothermia in mice (Oishi et al., 2013).

On the contrary, Genzer et al. (2015) fed mice with a LF diet or KD for 8 weeks, and observed that clock genes were phasedelayed under KD compared to the LF diet in the brain and the liver. Moreover, the amplitude of the circadian rhythm of clock genes in the liver was sixfold higher in the KD group, while their amplitude was lower in the brain, except for Bmal1 that showed an increase in the amplitude of oscillation. The high-amplitude circadian rhythm in the periphery reflected an increased locomotor activity. In contrast to Oishi, their gene analysis was performed under conditions of total darkness, thereby avoiding light as a confounding factor. 
Tognini et al. (2017) studied the molecular mechanisms which may lay behind the influence of $\mathrm{KD}$ on circadian rhythm. The authors fed mice ad libitum with normal chow or a KD for 4 weeks, and then studied the effects of the diets in the liver and the gut. With normal chow, the number of cyclic genes was similar in the liver and in the gut, while KD induced many de novo oscillating genes in the liver, and reduced the oscillating genes in the gut, revealing a tissue-specificity of the metabolic effects induced by the KD. Moreover, the diurnal KD-induced genes oscillated in a coordinated manner in the liver and in the gut. Among the genes oscillating both in normal chow and $\mathrm{KD}$ diet, more than a half had an increased amplitude in the liver under the KD diet. The expression of hepatic and gut core clock genes did not differ in mice fed the $\mathrm{KD}$ in comparison to normal chow-mice, so that core clock genes seem to be resistant to alterations induced by a food challenge. KD modulates the clock machinery recruitment to chromatin, leading to changes in the coupling of the core molecular clock machinery to output pathways. This is different to the HF diet which tends to hinder the chromatin recruitment of Bmal1 (Eckel-Mahan et al., 2013). The ketone, $\beta \mathrm{OHB}$, has an epigenetic role in histone posttranslational modifications.

In humans there are no studies on the effects of a ketogenic diet on the circadian rhythm. Anyway sleep, as discussed above, is a direct expression of circadian rhythm. In the literature, there are some indirect data on the effects of $\mathrm{KD}$ on sleep. Diet composition can influence sleep quality and structure (Zadeh and Begum, 2011; Santana et al., 2012; Grandner et al., 2013; Tanaka et al., 2013; Yamaguchi et al., 2013; Katagiri et al., 2014; St-Onge et al., 2016a,b; Zhou et al., 2016; Komada et al., 2017). Studies on patients treated with KD show some interesting results on improving sleep structure. A study of 11 epileptic children administered KD for 12 months (Hallbook et al., 2007) showed a reduction of total sleep and daytime sleep, intact slow-wave sleep and an increase in rapid eye movement (REM) sleep, associated with improved attentional behavior. Similarly, morbidly obese adolescents which were administered a highprotein, low-carbohydrate, low-fat KD experienced increased REM and decreases slow-wave sleep from a supraphysiological level (Willi et al., 1998).

Afaghi et al. (2008) studied a sample of 14 healthy non-obese men administered a very low carbohydrate diet which induced ketosis, and an isocaloric control mixed diet, and observed increased slow-wave sleep and decreased REM sleep during the very low-carbohydrate diet administration. Castro et al. (2018) recently studied the effect of a very low-calorie, ketogenic diet on sleep in 20 obese patients, measuring the diurnal sleep propensity with the Epworth Daytime Sleepiness Scale (ESS) and the quality of sleep with the questionnaire Pittsburgh Sleep Quality Index (PSQI). The quantity and quality of sleep were

\section{REFERENCES}

Afaghi, A., O'Connor, H., and Chow, C. M. (2008). Acute effects of the very low carbohydrate diet on sleep indices. Nutr. Neurosci. 11, 146-154. doi: 10.1179/ $147683008 \times 301540$ reported as not changed during the administration of diet, over a time of 12 weeks, but the reported sleepiness was reduced, suggesting a modification in sleep patterns, even if unrecognized by the patients.

\section{CONCLUDING REMARKS AND FUTURE PERSPECTIVES}

Circadian rhythms lie at the base of complex life, and are required for the regulation of energy metabolic processes. When peripheral clocks are led out of phase respect to the central clock, or among each other, metabolic alterations may occur. The timing of food intake, diet composition and sleep all play roles in regulating circadian rhythm.

Some research studies in animal models suggest that a ketogenic diet is able to influence circadian biology, through the modulation of clock gene expression. KD seems to have a profound impact on circadian rhythm, metabolism and behavioral activity, and induces a higher amplitude of gene expression and de novo oscillating genes at hepatic level. How these effects manifest, and what the long-term consequences are, remain to be determined. The role of ketosis itself is yet to be completely elucidated. At the same time, the effects of diet composition that have been studied and observed are mainly related to peripheral circadian clock regulation rather than central core clock regulation, so it is still not elucidated if nutrients may exert a direct effect on the central core clock rhythm.

Other studies with animal models and studies on circadian rhythm in humans are necessary to answer these questions which are recently born.

The interaction among diet, in particular the ketogenic diet, and circadian rhythm is extremely complex, and research at its dawn has already given intriguing results, taking potential important new horizons on obesity treatment.

\section{AUTHOR CONTRIBUTIONS}

EG and LG: conceptualization. EG: writing-original draft preparation. LG, AL, and DR: writing-review and editing. All authors have read and agreed to the published version of the manuscript.

\section{FUNDING}

DR would like to acknowledge the MRC program grant MR/P023576/1 and Wellcome Trust (107849/Z/15/Z).

Albrecht, U. (2012). Timing to perfection: the biology of central and peripheral circadian clocks. Neuron 74, 246-260. doi: 10.1016/j.neuron.2012.0 4.006

Almoosawi, S., Palla, L., Walshe, I., Vingeliene, S., and Ellis, J. G. (2018). Long sleep duration and social jetlag are associated inversely with a healthy dietary 
pattern in adults: results from the UK national diet and nutrition survey rolling programme $1^{-}$4. Nutrients 10:1131. doi: 10.3390/nu10091131

Bae, S. A., Fang, M. Z., Rustgi, V., Zarbl, H., and Androulakis, I. P. (2019). At the interface of lifestyle, behavior, and circadian rhythms: metabolic implications. Front. Nutr. 6:132. doi: 10.3389/fnut.2019.00132

Barnea, M., Madar, Z., and Froy, O. (2009). High-fat diet delays and fasting advances the circadian expression of adiponectin signaling components in mouse liver. Endocrinology 150, 161-168. doi: 10.1210/en.2008-0944

Barnea, M., Madar, Z., and Froy, O. (2010). High-fat diet followed by fasting disrupts circadian expression of adiponectin signaling pathway in muscle and adipose tissue. Obesity 18, 230-238. doi: 10.1038/oby.2009.276

Cain, S., Filtness, A., Phillips, C., and Anderson, C. (2015). Enhanced preference for high-fat foods following a simulated night shift. Scand. J. Work Environ. Health 41, 288-293. doi: 10.5271/sjweh.3486

Caprio, M., Infante, M., Moriconi, E., Armani, A., Fabbri, A., Mantovani, G., et al. (2019). Very-low-calorie ketogenic diet (VLCKD) in the management of metabolic diseases: systematic review and consensus statement from the Italian society of endocrinology (SIE). J. Endocrinol. Invest. 42, 1365-1386. doi: 10.1007/s40618-019-01061-2

Castro, A. I., Gomez-Arbelaez, D., Crujeiras, A. B., Granero, R., Aguera, Z., Jimenez-Murcia, S., et al. (2018). Effect of a very low-calorie ketogenic diet on food and alcohol cravings, physical and sexual activity, sleep disturbances, and quality of life in obese patients. Nutrients 10:1348. doi: 10.3390/nu10101348

Chaix, A., and Panda, S. (2016). Ketone bodies signal opportunistic food-seeking activity. Trends Endocrinol. Metab. 27, 350-352. doi: 10.1016/j.tem.2016.03.014

Chaput, J. P., Bouchard, C., and Tremblay, A. (2014). Change in sleep duration and visceral fat accumulation over 6 years in adults. Obesity 22, E9-E12.

Chavan, R., Feillet, C., Costa, S. S. F., Delorme, J. E., Okabe, T., Ripperger, J. A., et al. (2016). Liver-derived ketone bodies are necessary for food anticipation. Nat. Commun. 7:10580. doi: 10.1038/ncomms 10580

Chung, H., and Park, Y. K. (2017). Rationale, feasibility and acceptability of ketogenic diet for cancer treatment. J. Cancer Prev. 22, 127-134. doi: 10.15430/ JCP.2017.22.3.127

Damiola, F., Le Minh, N., Preitner, N., Kornmann, B., Fleury-olela, F., and Schibler, U. (2000). Restricted feeding uncouples circadian oscillators in peripheral tissues from the central pacemaker in the suprachiasmatic nucleus. Genes. Dev. 14, 2950-2961. doi: 10.1101/gad.183500

Dashti, H. S., Gómez-abellán, P., Qian, J., Esteban, A., Morales, E., and Scheer, F. A. J. L. (2021). Late eating is associated with cardiometabolic risk traits, obesogenic behaviors, and impaired weight loss. Am. J. Clin. Nutr. 113, 154-161. doi: $10.1093 /$ ajcn/nqaa264

Di Lorenzo, C., Pinto, A., Ienca, R., Coppola, G., Sirianni, G., Di Lorenzo, G., et al. (2019). A randomized double-blind, cross-over trial of very low-calorie diet in overweight migraine patients: a possible role for ketones? Nutrients 11:1742. doi: $10.3390 /$ nu11081742

Dibner, C., Schibler, U., and Albrecht, U. (2010). The mammalian circadian timing system?: organization and coordination of central and peripheral clocks. Annu. Rev. Physiol. 72, 517-549. doi: 10.1146/annurev-physiol-021909-135821

Eckel-Mahan, K. L., Patel, V. R., De Mateo, S., Orozco-Solis, R., Ceglia, N. J., Sahar, S., et al. (2013). Reprogramming of the circadian clock by nutritional challenge. Cell 155, 1464-1478. doi: 10.1016/j.cell.2013.11.034

Froy, O., Chapnik, N., and Miskin, R. (2009). Effect of intermittent fasting on circadian rhythms in mice depends on feeding time. Mech. Ageing Dev. 130, 154-160.

Gangitano, E., Tozzi, R., Gandini, O., Watanabe, M., Basciani, S., Mariani, S., et al. (2021). Ketogenic diet as a preventive and supportive care for COVID-19 patients. Nutrients 13:1004.

Genzer, Y., Dadon, M., Burg, C., Chapnik, N., and Froy, O. (2015). Ketogenic diet delays the phase of circadian rhythms and does not affect AMP-activated protein kinase (AMPK) in mouse liver. Mol. Cell. Endocrinol. 417, 124-130. doi: 10.1016/j.mce.2015.09.012

Grandner, M. A., Jackson, N., Gerstner, J. R., and Knutson, K. L. (2013). Dietary nutrients associated with short and long sleep duration. ata from a nationally representative sample. Appetite 64, 71-80. doi: 10.1016/j.appet.2013.01.004

Grant, C. L., Coates, A. M., Dorrian, J., Kennaway, D. J., Wittert, G. A., Heilbronn, L. K., et al. (2017). Timing of food intake during simulated night shift impacts glucose metabolism: a controlled study. Chronobiol. Int. 34, 1003-1013. doi: 10.1080/07420528.2017.1335318
Hallbook, T., Lundgren, J., Rosen, I., Hallböök, T., Lundgren, J., and Rosén, I. (2007). Ketogenic diet improves sleep quality in children with therapy-resistant epilepsy. Epilepsia 48, 59-65.

Hatori, M., Vollmers, C., Zarrinpar, A., Ditacchio, L., Bushong, E. A., Gill, S., et al. (2012). Time-restricted feeding without reducing caloric intake prevents metabolic diseases in mice fed a high-fat diet. Cell Metab. 15, 848-860.

Jakubowicz, D., Wainstein, J., Landau, Z., Raz, I., Ahren, B., Chapnik, N., et al. (2017). Influences of breakfast on clock gene expression and postprandial glycemia in healthy individuals and individuals with diabetes: a randomized clinical trial. Diabetes Care 40, 1573-1579. doi: 10.2337/dc16-2753

Katagiri, R., Asakura, K., Kobayashi, S., Suga, H., and Sasaki, S. (2014). Low intake of vegetables, high intake of confectionary, and unhealthy eating habits are associated with poor sleep quality among middle-aged female Japanese workers. J. Occup. Health 56, 359-368. doi: 10.1539/joh.14-0051-oa

Kennaway, D. J., Owens, J. A., Voultsios, A., Boden, M. J., and Varcoe, T. J. (2007). Metabolic homeostasis in mice with disrupted clock gene expression in peripheral tissues. Am. J. Physiol. Regul. Integr. Comp. Physiol. 293, R1528R1537.

Kimura, I., Inoue, D., Maeda, T., Hara, T., Ichimura, A., Miyauchi, S., et al. (2011). Short-chain fatty acids and ketones directly regulate sympathetic nervous system via G protein-coupled receptor 41 (GPR41). Proc. Natl. Acad. Sci. U.S.A. 108, 8030-8035. doi: 10.1073/pnas.1016088108

Kirkpatrick, C. F., Bolick, J. P., Kris-Etherton, P. M., Sikand, G., Aspry, K. E., Soffer, D. E., et al. (2019). Review of current evidence and clinical recommendations on the effects of low-carbohydrate and very-low-carbohydrate (including ketogenic) diets for the management of body weight and other cardiometabolic risk factors: a scientific statement from the national lipid association nutrition and lifestyle task force. J. Clin. Lipidol. 13, 689.e-711.e. doi: 10.1016/j.jacl.2019. 08.003

Ko, C. H., and Takahashi, J. S. (2006). Molecular components of the mammalian circadian clock. Hum. Mol. Genet. 15, R271-R277.

Kohsaka, A., Laposky, A. D., Ramsey, K. M., Estrada, C., Joshu, C., Kobayashi, Y., et al. (2007). High-fat diet disrupts behavioral and molecular circadian rhythms in mice. Cell Metab. 6, 414-421.

Kolbe, I., Leinweber, B., Brandenburger, M., and Oster, H. (2019). Circadian clock network desynchrony promotes weight gain and alters glucose homeostasis in mice. Mol. Metab. 30, 140-151. doi: 10.1016/j.molmet.2019.09.012

Komada, Y., Narisawa, H., Ueda, F., Saito, H., Sakaguchi, H., Mitarai, M., et al. (2017). Relationship between self-reported dietary nutrient intake and selfreported sleep duration among Japanese adults. Nutrients 9:134. doi: 10.3390/ nu9020134

Mathew, G. M., Hale, L., and Chang, A.-M. (2020). Social jetlag, eating behaviours, and body mass index among adolescents in the USA. Br. J. Nutr. 124, 979-987. doi: $10.1017 /$ S0007114520001804

McHill, A. W., and Wright, K. P. (2017). Role of sleep and circadian disruption on energy expenditure and in metabolic predisposition to human obesity and metabolic disease. Obes. Rev. 18 (Suppl. 1), 15-24. doi: 10.1111/obr.12503

Mchill, A. W., Czeisler, C. A., Phillips, A. J. K., Keating, L., Barger, L. K., Garaulet, M., et al. (2019). Caloric and macronutrient intake differ with circadian phase and between lean and overweight young adults. Nutrients 11:587. doi: 10.3390/ nu11030587

Mchill, A. W., Phillips, A. J. K., Czeisler, C. A., Keating, L., Yee, K., Barger, L. K., et al. (2017). Later circadian timing of food intake is associated with increased body fat. Am. J. Clin. Nutr. 106, 1213-1219.

Mezick, E. J., Wing, R. R., and McCaffery, J. M. (2014). Associations of selfreported and actigraphy-assessed sleep characteristics with body mass index and waist circumference in adults: moderation by gender. Sleep Med. 15, 64-70. doi: 10.1016/j.sleep.2013.08.784

Newman, J. C., and Verdin, E. (2014). Ketone bodies as signaling metabolites. Trends Endocrinol. Metab. 25, 42-52. doi: 10.1016/j.tem.2013.09.002

Oishi, K., Uchida, D., Ohkura, N., Doi, R., Ishida, N., Kadota, K., et al. (2009). Ketogenic diet disrupts the circadian clock and increases hypofibrinolytic risk by inducing expression of plasminogen activator inhibitor-1. Arter. Thromb. Vasc. Biol. 29, 1571-1577. doi: 10.1161/ATVBAHA.109.190140

Oishi, K., Yamamoto, S., Uchida, D., and Doi, R. (2013). Ketogenic diet and fasting induce the expression of cold-inducible RNA-binding protein with timedependent hypothermia in the mouse liver. FEBS Open Bio 3, 192-195. doi: 10.1016/j.fob.2013.03.005 
Panda, S. (2016). Circadian physiology of metabolism. Science. 354, 1008-1015. doi: 10.1126/science.aah4967

Paoli, A., Mancin, L., Giacona, M. C., Bianco, A., and Caprio, M. (2020). Effects of a ketogenic diet in overweight women with polycystic ovary syndrome. J. Transl. Med. 18:104. doi: 10.1186/s12967-020-02277-0

Paoli, A., Rubini, A., Volek, J. S., and Grimaldi, K. A. (2013). Beyond weight loss: a review of the therapeutic uses of very-low-carbohydrate (ketogenic) diets. Eur. J. Clin. Nutr. 67, 789-796. doi: 10.1038/ejcn.2013.116

Poggiogalle, E., Jamshed, H., and Peterson, C. M. (2018). Circadian regulation of glucose, lipid, and energy metabolism in humans. Metabolism 84, 11-27. doi: 10.1016/j.metabol.2017.11.017

Potter, G. D. M., Cade, J. E., and Hardie, L. J. (2017). Longer sleep is associated with lower BMI and favorable metabolic profiles in UK adults: findings from the National diet and nutrition survey. PLoS One 12:e0182195. doi: 10.1371/ journal.pone. 0182195

Reinke, H., and Asher, G. (2019). Crosstalk between metabolism and circadian clocks. Nat. Rev. Mol. Cell Biol. 20, 227-241. doi: 10.1038/s41580-018-0 096-9

Salgado-Delgado, R. C., Saderi, N., Basualdo Mdel, C., Guerrero-Vargas, N. N., Escobar, C., and Buijs, R. M. (2013). Shift work or food intake during the rest phase promotes metabolic disruption and desynchrony of liver genes in male rats. PLoS One 8:e60052 doi: 10.1371/journal.pone.006 0052

Salgado-delgado, R., Angeles-castellanos, M., Saderi, N., Buijs, R. M., and Escobar, C. (2010). Food intake during the normal activity phase prevents obesity and circadian desynchrony in a rat model of night work. Endocrinology 151, 1019-1029. doi: 10.1210/en.2009-0864

Santana, A. A., Pimentel, G. D., Romualdo, M., Oyama, L. M., Santos, R. V., Pinho, R. A., et al. (2012). Sleep duration in elderly obese patients correlated negatively with intake fatty. Lipids Health Dis. 11:99.

Shimazu, T., Hirschey, M. D., Newman, J., He, W., Shirakawa, K., and Moan, N. (2013). Suppression of oxidative stress by $\beta$-hydroxybutyrate, an endogenous histone deacetylase inhibitor. Science 339, 211-215. doi: 10.1126/science. 1227166

Simon, S. L., Field, J., Miller, L. E., DiFrancesco, M., and Beebe, D. W. (2015). Sweet/dessert foods are more appealing to adolescents after sleep restriction. PLoS One 10:e0115434. doi: 10.1371/journal.pone.0115434

St-Onge, M.-P., Mikic, A., and Pietrolungo, C. E. (2016a). Effects of diet on sleep quality. Adv. Nutr. 7, 938-949.

St-Onge, M.-P., Roberts, A., Shechter, A., and Choudhury, A. R. (2016b). Fiber and saturated fat are associated with sleep arousals and slow wave sleep. J. Clin. Sleep Med. 12, 19-24. doi: 10.5664/jcsm.5384

Sukkar, S., Cogorno, L., Pisciotta, L., Pasta, A., Vena, A., Gradaschi, R., et al. (2021). Clinical efficacy of eucaloric ketogenic nutrition in the COVID-19 cytokine storm (CSS): a retrospective analysis of mortality and intensive care unit admission. Nutrition 89:111236. doi: 10.1016/j.nut.2021.111236

Taggart, A. K. P., Kero, J., Gan, X., Cai, T., Cheng, K., Ippolito, M., et al. (2005). (D)$\beta$-Hydroxybutyrate inhibits adipocyte lipolysis via the nicotinic acid receptor PUMA-G. J. Biol. Chem. 280, 26649-26652. doi: 10.1074/jbc.C500213200

Tanaka, E., Yatsuya, H., Uemura, M., Murata, C., Otsuka, R., Toyoshima, H., et al. (2013). Associations of protein, fat, and carbohydrate intakes with insomnia symptoms among middle-aged Japanese workers. J. epidemiol. 23, 132-138. doi: 10.2188/jea.je20120101
Tognini, P., Murakami, M., Liu, Y., Eckel-Mahan, K. L., Newman, J. C., Verdin, E., et al. (2017). Distinct circadian signatures in liver and gut clocks revealed by Ketogenic diet. Cell Metab. 26, 523.e-538.e. doi: 10.1016/j.cmet.2017.08.015

Turek, F. W., Joshu, C., Kohsaka, A., Lin, E., Ivanova, G., Laposky, A., et al. (2005). Obesity and metabolic syndrome in circadian clock mutant mice. Science 308 , 1043-1045.

Van Den Berg, J. F., Knvistingh Neven, A., Tulen, J. H. M., Hofman, A., Witteman, J. C. M., Miedema, H. M. E., et al. (2008). Actigraphic sleep duration and fragmentation are related to obesity in the elderly: the rotterdam study. Int. J. Obes. 32, 1083-1090. doi: 10.1038/ijo.2008.57

Willi, S. M., Oexmann, M. J., Wright, N. M., Collop, N. A., and Key, L. L. (1998). The effects of a high-protein, low-fat, ketogenic diet on adolescents with morbid obesity: body composition, blood chemistries, and sleep abnormalities. Pediatrics 101, 61-67. doi: 10.1542/peds.101.1.61

Wlodarek, D. (2019). Role of ketogenic diets in neurodegenerative diseases (Alzheimer's disease and Parkinson's Disease). Nutrients 11:169. doi: 10.3390/ nu11010169

$\mathrm{Wu}, \mathrm{T}$., Ni, Y., Zhuge, F., and Fu, Z. (2010). Resetting process of peripheral circadian gene expression after the combined reversal of feeding schedule and light/ dark cycle via a 24-h light period transition in rats. Physiol. Res. 59, 581-590. doi: 10.33549/physiolres.931818

Yamaguchi, M., Uemura, H., Katsuura-Kamano, S., Nakamoto, M., Hiyoshi, M., Takami, H., et al. (2013). Relationship of dietary factors and habits with sleep-wake regularity. Asia Pac. J. Clin. Nutr. 22, 457-465.

Yasumoto, Y., Hashimoto, C., Nakao, R., Yamazaki, H., Hiroyama, H., Nemoto, T., et al. (2016). Short-term feeding at the wrong time is sufficient to desynchronize peripheral clocks and induce obesity with hyperphagia, physical inactivity and metabolic disorders in mice. Metabolism 65, 714-727. doi: 10.1016/j.metabol. 2016.02.003

Zadeh, S. S., and Begum, K. (2011). Comparison of nutrient intake by sleep status in selected adults in Mysore, India. Nutr. Res. Pract. 5, 230-235. doi: 10.4162/ nrp.2011.5.3.230

Zhou, J., Kim, J. E., Armstrong, C. L. H., Chen, N., and Campbell, W. W. (2016). Higher-protein diets improve indexes of sleep in energy-restricted overweight and obese adults: results from 2 randomized controlled trials. Am. J. Clin. Nutr. 103, 766-774. doi: 10.3945/ajcn.115.124669

Conflict of Interest: The authors declare that the research was conducted in the absence of any commercial or financial relationships that could be construed as a potential conflict of interest.

Publisher's Note: All claims expressed in this article are solely those of the authors and do not necessarily represent those of their affiliated organizations, or those of the publisher, the editors and the reviewers. Any product that may be evaluated in this article, or claim that may be made by its manufacturer, is not guaranteed or endorsed by the publisher.

Copyright $(2021$ Gangitano, Gnessi, Lenzi and Ray. This is an open-access article distributed under the terms of the Creative Commons Attribution License (CC BY). The use, distribution or reproduction in other forums is permitted, provided the original author(s) and the copyright owner(s) are credited and that the original publication in this journal is cited, in accordance with accepted academic practice. No use, distribution or reproduction is permitted which does not comply with these terms. 